\title{
The MAP/G/1 G-queue with Unreliable Server and Multiple Vacations
}

Yi Peng

School of Mathematical Science, Changsha Normal University, Changsha 410100, Hunan, P.R. China

E-mail: scgyp06@163.com

Keywords: G-queues, Markovian Arrival Process (MAP), reliability, censoring technique, RG-factorization

Received: November 21, 2018

\begin{abstract}
In this paper, we consider a MAP/G/l G-queues with unreliable server and multiple vacations. The arrival of a negative customer not only removes the customer being in service, but also makes the server under repair. The server leaves for a vacation as soon as the system empties and is allowed to take repeated (multiple) vacations. By using the supplementary variables method and the censoring technique, we obtain the queue length distributions. We derive the mean of the busy period based on the renewal theory. Furthermore, we analyze some main reliability indexes and investigate some important special cases.

Povzetek: Predstavljena je nova metoda obravnave strežniških vrst v pogojih nezanesljivega delovanja in $v$ primerih občasne odsotnosti obdelave.
\end{abstract}

\section{Introduction}

Recently there has been a rapid increase in the literature on queueing systems with negative arrivals. Queues with negative arrivals, called G-queues, were first introduced by Gelenbe [1]. When a negative customer arrives at the queue, it immediately removes one or more positive customers if present. Negative arrivals have been interpreted as viruses, orders of demand, inhibiter. Queueing systems with negative arrivals have many applications in computer, neural networks, manufacturing systems and communication networks etc. There is a lot of research on queueing system with negatives arrivals. For a comprehensive survey on queueing systems with negative arrivals, readers may see [1-4].

Boucherie and Boxma [3] considered an M/G/1 queue with negative arrivals where a negative arrival removes a random amount of work. Li and Zhao [5] discussed an MAP/G/1 queue with negative arrivals. They analyzed two classes of removal rules: (i) arrival of a negative customer which removes all the customers in the system (RCA); (ii) arrival of a negative customer which removes only a customer from the head of the system $(\mathrm{RCH})$, including the customer being in service.

Queueing system with repairable server has been studied by many authors such as Cao and Chen [6], Neuts and Lucantoni [7]. Wang, Cao and $\mathrm{Li}[8]$ analyzed the reliability of the retrial queues with server breakdowns and repairs. Harrison and Pitel [9]considered the M/M/1 G-queues with breakdowns and exponential repair times. $\mathrm{Li}$, Ying and Zhao [10] investigated a BMAP/G/1 retrial queue with a server subject to breakdowns and repairs.

For a detailed survey on queueing systems with server vacations one can refer to Refs [11]. Recently, Sikdar and Gupta [12] discussed the queue length distributions in the finite buffer bulk-service MAP/G/1 queue with multiple vacations. Kasahara, Takine, Takahashi and Hasegawa [13] considered the MAP/G/1 queues under N-policy with and without vacations.

Most of the analysis in the past have been carried out assuming Poisson input. However, in recent years there has been a growing interest to analyze queues by considering input process as Markovian arrival process (MAP). The MAP is a useful mathematical model for describing bursty traffic in modern communication networks, and is a rich class of point processes containing many familiar arrival processes such as Poission process, PH-renewal process, Markov modulated Poission process, etc. Readers may refer to chapter 8 in Bocharov [14].

In this paper, we consider the MAP/G/1 G-queues with unreliable server and multiple vacations. The process of arrivals of negative customers is also MAP. The arrival of a negative customer not only removes the customer being in service, but also makes the server under repair. We obtain the distributions of stationary queue length, the mean of the busy period and some reliability indexes by using the supplementary variable method, the matrixanalytic method, the censoring technique, and the renewal theory.

The rest of this paper is organized as follows. The model description is given in section 2 . The stationary differential equations of the model and their solutions are obtained in section 3 . The expressions for the distributions of the stationary queue length and the mean of the busy period are derived in section 4. Some special cases are considered in section 5. Some numerical examples are shown in section 6 . 


\section{Model description}

In this section, we consider a single server queue with two types of independent arrivals, positive and negative. Positive arrivals correspond to customers who upon arrival, join the queue with the intention of being served and then leaving the system. At a negative arrival epoch, the system is affected if and only if the server is working.

The arrival process. We assume that the arrivals of both positive and negative customers are MAPs with matrix descriptors $\left(C_{1}, D_{1}\right)$ and $\left(C_{2}, D_{2}\right)$ respectively, where the infinitesimal generators $C_{1}+D_{1}$ and $C_{2}+D_{2}$ of sizes $m_{1} \times m_{1}$ and $m_{2} \times m_{2}$, respectively, are irreducible and positive recurrent. Let $\theta_{1}$ and $\theta_{2}$ be the stationary probability vectors of $C_{1}+D_{1}$ and $C_{2}+D_{2}$, respectively. Then $\lambda_{1}=\theta_{1} D_{1} e$ and $\lambda_{2}=\theta_{2} D_{2} e$ are the stationary arrival rates of positive and negative customers, respectively, where is a column vector of ones of a suitable size.

The removal rule. The arrival of a negative customer not only removes the customer being in service, but also makes the server under repair. And after repair the server is as good as new. As soon as the repair of the server is completed, the server enters the working state immediately and continues to serve the next customer if the queue is not empty.

The vacations. When the server finishes serving a positive customer or the repair of the server is completed and finds the queue empty, the server leaves for a vacation of random length V. On return from a vacation if he finds more than one customer waiting, he takes the customer from the head of the queue for service and continues to serve in this manner until the queue is empty. Otherwise, he immediately goes for another vacation.

The service time. All positive customers have i.i.d. service time distribution given by

[Beginning of the document] [Automatic

$$
B_{S}(x)=1-\exp \left\{-\int_{0}^{x} \mu_{S}(\nu) d \nu\right\}
$$
break]

mean $1 / \mu_{S} \in(0,+\infty)$.

The vacation time. The vacation time distribution is given by

$$
B_{V}(x)=1-\exp \left\{-\int_{0}^{x} \mu_{V}(\nu) d \nu\right\}
$$
with mean $1 / \mu_{V} \in(0,+\infty)$.

The repair time. The repair time distribution is given by

$$
B_{R}(x)=1-\exp \left\{-\int_{0}^{x} \mu_{R}(\nu) d \nu\right\}
$$

with mean $1 / \mu_{R} \in(0,+\infty)$.The independence. We assume that all the random variables defined above are independent. Throughout the rest of the paper, we denote by $\bar{F}(x)=1-F(x)$ the tail of distribution function $F(x)$.

\section{The differential equations and the solution}

In this section, we first introduce several supplementary variables to construct the differential equations for the model. We then use the censoring technique to solve these equations. The solution to the differential equations will be used to obtain interesting performance measures of the system in later sections.

Let $N(t)$ be the number of customers in the system at time $t$, and let $J_{1}(t)$ and $J_{2}(t)$ be the phases of the arrivals of positive and negative customers at time ${ }^{t}$, respectively. We define the states of the server as

$I(t)= \begin{cases}S, & \text { if the server is working with service time distribution } B_{S}(x), \\ V, & \text { if the server is on vacation with vacation time distribution } B_{V}(x),\end{cases}$

$R$, if the server is being repaired with repair time distribution $B_{R}(x)$.

For $t>0$, we define the random variable $S(t)$ as follows:(i) if $I(t)=S, S(t)$ represents the elapsed service time received by a customer with the service time up to time ${ }^{t}$; (ii)if $I(t)=V, S(t)$ represents the elapsed vacation time up to time $t$; (iii) if $I(t)=R, S(t)$ represents the elapsed repair time up to time $t$. Then, $\left\{I(t), N(t), J_{1}(t), J_{2}(t), S(t): t \geq 0\right\}$ is a Markov process. The state space of the process is expressed as

$\Omega=\left\{\left(S, k, j_{1}, j_{2}, x\right): k \geq 1,1 \leq j_{1} \leq m_{1}, 1 \leq j_{2} \leq m_{2}, x \geq 0\right\}$

$\cup\left\{\left(V, k, j_{1}, j_{2}, x\right): k \geq 0,1 \leq j_{1} \leq m_{1}, 1 \leq j_{2} \leq m_{2}, x \geq 0\right\}$

$\cup\left\{\left(R, k, j_{1}, j_{2}, x\right): k \geq 0,1 \leq j_{1} \leq m_{1}, 1 \leq j_{2} \leq m_{2}, x \geq 0\right\}$

We write:

$p_{S, k, i, j}(t, x) d x=p\left\{I(t)=S, N(t)=k, J_{1}(t)=i, J_{2}(t)=j, x \leq S(t)<x+d x\right\}$, $p_{V, k, i, j}(t, x) d x=p\left\{I(t)=V, N(t)=k, J_{1}(t)=i, J_{2}(t)=j, x \leq S(t)<x+d x\right\}$, $p_{R, k, i, j}(t, x) d x=p\left\{I(t)=R, N(t)=k, J_{1}(t)=i, J_{2}(t)=j, x \leq S(t)<x+d x\right\}$, $p_{S, k, i, j}(x)=\lim _{t \rightarrow+\infty} p_{S, k, i, j}(t, x)$

$p_{V, k, i, j}(x)=\lim _{t \rightarrow+\infty} p_{V, k, i, j}(t, x)$,

$p_{R, k, i, j}(x)=\lim _{t \rightarrow+\infty} p_{R, k, i, j}(t, x)$,

$P_{k}^{S}(x)=\left(p_{S, k, 1,1}(x), \cdots, p_{S, k, 1, m_{2}}(x), \cdots, p_{S, k, m_{1}, 1}(x), \cdots, p_{S, k, m_{1}, m_{2}}(x)\right)$, $P_{k}^{V}(x)=\left(p_{V, k, 1,1}(x), \cdots, p_{V, k, 1, m_{2}}(x), \cdots, p_{V, k, m_{1}, 1}(x), \cdots, p_{V, k, m_{1}, m_{2}}(x)\right)$, $P_{k}^{R}(x)=\left(p_{R, k, 1,1}(x), \cdots, p_{R, k, 1, m_{2}}(x), \cdots, p_{R, k, m_{1}, 1}(x), \cdots, p_{R, k, m_{1}, m_{2}}(x)\right)$.

If the system is stable, then the system of stationary differential equations of the joint probability density $\left\{P_{0}^{V}(x), P_{0}^{R}(x), P_{k}^{S}(x), P_{k}^{V}(x), P_{k}^{R}(x), k \geq 1\right\}$ can be written as

$\frac{d}{d x} P_{1}^{S}(x)=P_{1}^{S}(x)\left[C_{1} \oplus C_{2}-\mu_{S}(x) I\right]$,

$\frac{d}{d x} P_{k}^{S}(x)=P_{k}^{S}(x)\left[C_{1} \oplus C_{2}-\mu_{S}(x) I\right]+P_{k-1}^{S}(x)\left(D_{1} \otimes I\right), k \geq 2$,

$\frac{d}{d x} P_{0}^{V}(x)=P_{0}^{V}(x)\left[C_{1} \oplus C_{2}+I \otimes D_{2}-\mu_{V}(x) I\right]$,

$\frac{d}{d x} P_{k}^{V}(x)=P_{k}^{V}(x)\left[C_{1} \oplus C_{2}+I \otimes D_{2}-\mu_{V}(x) I\right]+P_{k-1}^{V}(x)\left(D_{1} \otimes I\right), k \geq 1$ 
$\frac{d}{d x} P_{0}^{R}(x)=P_{0}^{R}(x)\left[C_{1} \oplus C_{2}+I \otimes D_{2}-\mu_{R}(x) I\right]$,

$\frac{d}{d x} P_{k}^{R}(x)=P_{k}^{R}(x)\left[C_{1} \oplus C_{2}+I \otimes D_{2}-\mu_{R}(x) I\right]+P_{k-1}^{R}(x)\left(D_{1} \otimes I\right), k \geq 1$.

The joint probability density $\left\{P_{0}^{V}(x), P_{0}^{R}(x), P_{k}^{S}(x), P_{k}^{V}(x), P_{k}^{R}(x), k \geq 1\right\}$ should satisfy the boundary conditions:

$P_{0}^{V}(0)=\int_{0}^{+\infty} P_{1}^{S}(x) \mu_{S}(x) d x+\int_{0}^{+\infty} P_{0}^{V}(x) \mu_{V}(x) d x+\int_{0}^{+\infty} P_{0}^{R}(x) \mu_{R}(x) d x$,

$P_{k}^{V}(0)=0, \quad k \geq 1$,

$P_{k}^{R}(0)=\int_{0}^{+\infty} P_{k+1}^{S}(x) d x\left(I \otimes D_{2}\right), \quad k \geq 0$,

$P_{k}^{S}(0)=\int_{0}^{+\infty} P_{k+1}^{S}(x) \mu_{S}(x) d x+\int_{0}^{+\infty} P_{k}^{V}(x) \mu_{V}(x) d x$ $+\int_{0}^{+\infty} P_{k}^{R}(x) \mu_{R}(x) d x \quad k \geq 1$,

and the normalization condition:

$\left\{\sum_{k=0}^{\infty} \int_{0}^{+\infty} P_{k}^{V}(x) d x+\sum_{k=0}^{\infty} \int_{0}^{+\infty} P_{k}^{R}(x) d x+\sum_{k=1}^{\infty} \int_{0}^{+\infty} P_{k}^{S}(x) d x\right\} e=1$.

In the remainder of this section, we solve equations (1)-(11). To sovle equations (1)-(6). we define $Q_{S}^{*}(z, x)=\sum_{k=1}^{\infty} z^{k} P_{k}^{S}(x), Q_{V}^{*}(z, x)=\sum_{k=0}^{\infty} z^{k} P_{k}^{V}(x), Q_{R}^{*}(z, x)=\sum_{k=0}^{\infty} z^{k} P_{k}^{R}(x)$.

It follows from (1) and (2) that $\frac{\partial}{\partial x} Q_{S}^{*}(z, x)=Q_{S}^{*}(z, x)\left[\left(C_{1}+z D_{1}\right) \oplus C_{2}-\mu_{S}(x) I\right]$, which leads to $Q_{S}^{*}(z, x)=Q_{S}^{*}(z, 0)\left[\exp \left\{\left(C_{1}+z D_{1}\right) x\right\} \otimes \exp \left\{C_{2} x\right\}\right] \overline{B_{S}}(x)$.

It follows from (3) and (4) that

$\frac{\partial}{\partial x} Q_{V}^{*}(z, x)=Q_{V}^{*}(z, x)\left[\left(C_{1}+z D_{1}\right) \oplus\left(C_{2}+D_{2}\right)-\mu_{V}(x) I\right]$, which leads to

$Q_{V}^{*}(z, x)=Q_{V}^{*}(z, 0)\left[\exp \left\{\left(C_{1}+z D_{1}\right) x\right\} \otimes \exp \left\{\left(C_{2}+D_{2}\right) x\right\}\right] \overline{B_{V}}(x)$.

It follows from (5) and (6) that

$\frac{\partial}{\partial x} Q_{R}^{*}(z, x)=Q_{R}^{*}(z, x)\left[\left(C_{1}+z D_{1}\right) \oplus\left(C_{2}+D_{2}\right)-\mu_{R}(x) I\right]$,

which leads to

$Q_{R}^{*}(z, x)=Q_{R}^{*}(z, 0)\left[\exp \left\{\left(C_{1}+z D_{1}\right) x\right\} \otimes \exp \left\{\left(C_{2}+D_{2}\right) x\right\}\right] \overline{B_{R}}(x)$

Let us define $P(n, t), n \geq 0, t \geq 0$ as $m_{1} \times m_{1}$ matrix whose element $(P(n, t))_{i j}$ is the probability that exactly ${ }^{n}$ positive customers arrive during $[0, t)$ and the generation process passes from phase $i$ to phase $j$. These matrices satisfy the following system of differential equations

$\frac{d}{d t} P(0, t)=P(0, t) C_{1}$,

$\frac{d}{d t} P(n, t)=P(n, t) C_{1}+P(n-1, t) D_{1}, \quad n \geq 1$,

with $P(0,0)=I \cdot$ We define

$P^{*}(z, t)=\sum_{n=0}^{\infty} z^{n} P(n, t), \quad|z| \leq 1$,

Solving the above matrix differential equation,we get $P^{*}(z, t)=e^{\left(C_{1}+z D_{1}\right) t}, \quad|z| \leq 1, t \geq 0$.

Substituting (15) into (12)-(14) respectively gives $P_{k}^{S}(x)=\sum_{j=1}^{k} P_{j}^{S}(0)\left[P(k-j, x) \otimes \exp \left\{C_{2} x\right\}\right] \overline{B_{S}}(x), k \geq 1$,
$P_{k}^{V}(x)=\sum_{j=0}^{k} P_{j}^{V}(0)\left[P(k-j, x) \otimes \exp \left\{\left(C_{2}+D_{2}\right) x\right\}\right] \overline{B_{V}}(x)$

$=P_{0}^{V}(0)\left[P(k, x) \otimes \exp \left\{\left(C_{2}+D_{2}\right) x\right\}\right] \overline{B_{V}}(x), \quad k \geq 0$.

$P_{k}^{R}(x)=\sum_{j=0}^{k} P_{j}^{R}(0)\left[P(k-j, x) \otimes \exp \left\{\left(C_{2}+D_{2}\right) x\right\}\right] \overline{B_{R}}(x), k \geq 0$,

Equations (16)-(18) provide a solution for the system of differential equations (1)-(6). Furthermore, boundary equations (7)-(10) will be used to determine the vectors $P_{k}^{S}(0)$ for $k \geq 1, P_{k}^{R}(0)$ for $k \geq 0$ and $P_{k}^{V}(0)$ for $k \geq 0$.We define:

$A_{k}=\int_{0}^{+\infty}\left[P(k, x) \otimes \exp \left\{C_{2} x\right\}\right] d B_{S}(x)$

$B_{k}=\int_{0}^{+\infty}\left[P(k, x) \otimes \exp \left\{\left(C_{2}+D_{2}\right) x\right\}\right] d B_{R}(x)$

$W_{k}=\int_{0}^{+\infty}\left[P(k, x) \otimes \exp \left\{\left(C_{2}+D_{2}\right) x\right\}\right] d B_{V}(x)$,

$E_{k}=\int_{0}^{+\infty}\left[P(k, x) \otimes \exp \left\{C_{2} x\right\}\right] \overline{B_{S}}(x) d x\left(I \otimes D_{2}\right)$

Then it follows from (7)-(10),(16)-(18)that $P=P \Pi$ , where

$P=\left(P_{0}^{V}(0), P_{1}^{S}(0), P_{0}^{R}(0), P_{2}^{S}(0), P_{1}^{R}(0), \cdots\right)$

and

$\Pi=\left(\begin{array}{ccccc}W_{0} & \widetilde{W_{1}} & \widetilde{W_{2}} & \widetilde{W_{3}} & \cdots \\ H_{0} & \widetilde{A_{1}} & \widetilde{A_{2}} & \widetilde{A_{3}} & \cdots \\ & \widetilde{A_{0}} & \widetilde{A_{1}} & \widetilde{A_{2}} & \cdots \\ & & \widetilde{A_{0}} & \widetilde{A_{1}} & \cdots \\ & & & \widetilde{A_{0}} & \cdots \\ & & & & \ddots\end{array}\right)$

With

$$
\widetilde{W_{k}}=\left(W_{k}, 0\right), \quad H_{0}=\left(\begin{array}{c}
A_{0} \\
B_{0}
\end{array}\right)
$$

$\widetilde{A_{k}}=\left(\begin{array}{cc}A_{k} & E_{k-1} \\ B_{k} & 0\end{array}\right), \quad E_{-1}=0, \quad k \geq 0$,

Therefore, we obtain the transition probability matrix and stationary differential equations of the system.

\section{Performance measures of the model}

In this section,we consider two performance measures for the model: the stationary queue length, the busy period.

\subsection{The stationary queue length}

We write

$p_{k}=\lim _{t \rightarrow \infty} P\{N(t)=k\}, \quad k \geq 0$,

$p_{k}^{S}=\lim _{t \rightarrow \infty} P\{N(t)=k, I(t)=S\}, \quad k \geq 1$,

$p_{k}^{V}=\lim _{t \rightarrow \infty} P\{N(t)=k, I(t)=V\}, \quad k \geq 0$,

$p_{k}^{R}=\lim _{t \rightarrow \infty} P\{N(t)=k, I(t)=R\}, \quad k \geq 0$.

Obviously,

$p_{0}=p_{0}^{V}+p_{0}^{R} ; \quad p_{k}=p_{k}^{S}+p_{k}^{V}+p_{k}^{R}, \quad k \geq 1$. 
Theorem 1 If the model is stable, then

$\left\{p_{0}=\beta\left(x_{0} H_{0}^{V}+x_{0}^{R} H_{0}^{R}\right) e\right.$,

$\left\{\begin{array}{l}p_{k}=\beta x_{0} H_{k}^{V} e+\beta \sum_{j=1}^{k} x_{j}^{S} H_{k-j}^{S} e+\beta \sum_{j=0}^{k} x_{j}^{R} H_{k-j}^{R} e, \quad k \geq 1,\end{array}\right.$

where

$H_{k}^{S}=\int_{0}^{+\infty}\left[P(k, x) \otimes \exp \left\{C_{2} x\right\}\right] \overline{B_{S}}(x) d x, \quad k \geq 0$,

$H_{k}^{V}=\int_{0}^{+\infty}\left[P(k, x) \otimes \exp \left\{\left(C_{2}+D_{2}\right) x\right\}\right] \overline{B_{V}}(x) d x, \quad k \geq 0$,

$H_{k}^{R}=\int_{0}^{+\infty}\left[P(k, x) \otimes \exp \left\{\left(C_{2}+D_{2}\right) x\right\}\right] \overline{B_{R}}(x) d x, \quad k \geq 0$,

So that the mean number of customers in the system is

$L=\sum_{k=0}^{\infty} k p_{k}=\beta x_{0} \sum_{k=1}^{\infty} k H_{k}^{V} e+\beta \sum_{k=1}^{\infty} k \sum_{j=1}^{k} x_{j}^{S} H_{k-j}^{S} e+\beta \sum_{k=1}^{\infty} k \sum_{j=0}^{k} x_{j}^{R} H_{k-j}^{R} e$.

Proof: It follows from (17) and (18) that

$p_{0}=\int_{0}^{+\infty}\left[P_{0}^{V}(x)+P_{0}^{R}(x)\right] d x e=\beta\left(x_{0} H_{0}^{V}+x_{0}^{R} H_{0}^{R}\right) e$,

and from (16)-(18) that

$p_{k}^{S}=\int_{0}^{+\infty} P_{k}^{S}(x) d x e=\beta \sum_{j=1}^{k} x_{j}^{S} H_{k-j}^{S} e$,

$p_{k}^{V}=\int_{0}^{+\infty} P_{k}^{V}(x) d x e=\beta x_{0} H_{k}^{V} e$,

$p_{k}^{R}=\int_{0}^{+\infty} P_{k}^{R}(x) d x e=\beta \sum_{j=0}^{k} x_{j}^{R} H_{k-j}^{R} e$.

This

completes the proof.

\subsection{The busy period}

We now provide an analysis of the busy period (including of the period when the server is under repair) of the model.

Let $\mathrm{V}$ be the random variable of the vacation time, or $B_{V}(x)=P\{V \leq x\}$.

We denote by $T$ be the random variable of the interarrival time between two positive customers, and $T^{(E)}$ the random variable for the equilibrium excess distributions with respect to $T$. Then we have $A(x)=P\{T \leq x\}=\theta_{1} \int_{0}^{x} \exp \left\{C_{1} t\right\} d t D_{1} e$

And

$A^{(E)}(x)=P\left\{T^{(E)} \leq x\right\}=\frac{1}{\theta_{1}\left(-C_{1}\right)^{-1} e} \int_{0}^{x} \bar{A}(t) d t$.

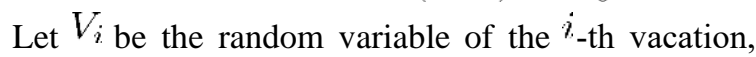
and $\hat{V}$ be the random variable of the number of times of vacations during the total vacation period. Then

$$
\begin{aligned}
P\{\hat{V}=n\} & =P\left\{\sum_{i=1}^{n-1} V_{i}<T^{(E)} \leq \sum_{i=1}^{n} V_{i}\right\} \\
& =\int_{0}^{+\infty}\left[B_{V}^{(n-1) *}(t)-B_{V}^{n *}(t)\right] d A^{(E)}(t),
\end{aligned}
$$

We denote by $F(x) * G(x)$ the convolution of two functions $F(x)$ and $G(x)$ given by $F(x) * G(x)=\int_{0}^{x} F(x-u) d G(u)$. We write
$F^{n *}(x)=F(x) * F^{(n-1) *}(x)$ for $n \geq 2$ and define $F^{0 *}(x)=1$.

Lemma 1 Let $\bar{V}$ be the random variable of the length of multiple vacations, then

$$
E \bar{V}=\sum_{n=1}^{\infty} n \frac{1}{\mu_{V}} \int_{0}^{+\infty}\left[B_{V}^{(n-1) *}(t)-B_{V}^{n *}(t)\right] d A^{(E)}(t) .
$$

Theorem 2 Let $\xi$ be the random variable of the busy period of the system, then

$E \xi=\frac{\left(1-\beta x_{0} L_{V} e\right) E \bar{V}}{\beta x_{0} L_{V} e}$.

Proof: According to the renewal theory, we can obtain $\sum_{k=0}^{\infty} p_{k}^{V}=\frac{E \bar{V}}{E \xi+E \bar{V}}$

$$
E \xi=\frac{\left(1-\sum_{k=0}^{\infty} p_{k}^{V}\right) E \bar{V}}{\sum_{k=0}^{\infty} p_{k}^{V}}=\frac{\left(1-\beta x_{0} L_{V} e\right) E \bar{V}}{\beta x_{0} L_{V} e} .
$$

or

This completes the proof.

Consequently, we obtain some important performance measures for the model: the stationary queue length, the mean number of customers in the system, the mean length of multiple vacations and the mean busy period.

\section{Special cases}

In this section we will investigate very briefly some important special cases.

Case 1. No negative arrival takes place and the server is reliable.

In this case, our model becomes the MAP/G/1 queue with multiple vacations.

We put $C_{2}=D_{2}=0$ and $B_{R}(x)=0$ in the main results and obtain

$Q=\left(\begin{array}{cccc}A_{1} & A_{2} & A_{3} & \cdots \\ A_{0} & A_{1} & A_{2} & \cdots \\ & A_{0} & A_{1} & \cdots \\ & & A_{0} & \cdots \\ & & & \ddots\end{array}\right)$

$A_{k}=\int_{0}^{+\infty} P(k, x) d B_{S}(x), \quad W_{k}=\int_{0}^{+\infty} P(k, x) d B_{V}(x)$,

$R_{j}=\sum_{i=1}^{\infty} A_{i+j} G_{1}^{i-1}\left[I-\Phi_{0}\right]^{-1}, j \geq 1, \quad R_{0, j}=\sum_{i=0}^{\infty} W_{i+j} G_{1}^{i}\left[I-\Phi_{0}\right]^{-1}, j \geq 1$,

$\Psi_{0}=W_{0}+\sum_{i=0}^{\infty} W_{i+1} G_{1}^{i}\left[I-\sum_{i=1}^{\infty} A_{i} G_{1}^{i-1}\right]^{-1} A_{0}, \quad G_{1}=\hat{Q}(1,1) A_{0}$,

$L_{V}=\int_{0}^{+\infty} \exp \left\{\left(C_{1}+D_{1}\right) x\right\} \overline{B_{V}}(x) d x, \quad L_{S}=\int_{0}^{+\infty} \exp \left\{\left(C_{1}+D_{1}\right) x\right\} \overline{B_{S}}(x) d x$,

$\left\{p_{0}=\beta x_{0} H_{0}^{V} e\right.$,

$\left\{p_{k}=\beta x_{0} H_{k}^{V} e+\beta \sum_{j=1}^{k} x_{j}^{S} H_{k-j}^{S} e, \quad k \geq 1\right.$, $H_{k}^{S}=\int_{0}^{+\infty} P(k, x) \overline{B_{S}}(x) d x, \quad H_{k}^{V}=\int_{0}^{+\infty} P(k, x) \overline{B_{V}}(x) d x, \quad k \geq 0$. 
Case 2. No vacation is allowed, in this case, our model becomes the MAP/G/1 G-queue with unreliable server.We assume that $B_{V}(x)=0$ in the main results and obtain $W_{0}=0, \quad \widetilde{W_{1}}=D_{1} \otimes I, \quad \widetilde{W_{k}}=0, \quad k \geq 2$, $R_{0,1}=\widetilde{W}_{1}\left[I-\Phi_{0}\right]^{-1}, \quad R_{0, j}=0, \quad j \geq 2$, $\beta=\frac{1}{x_{0} e+\sum_{k=1}^{\infty} x_{k}^{S} L_{S} e+\sum_{k=0}^{\infty} x_{k}^{R} L_{R} e}, \quad \Psi_{0}=\widetilde{W}_{1}\left[I-\sum_{i=1}^{\infty} \widetilde{A}_{i} G_{1}^{i-1}\right]^{-1} H_{0}$, $\left\{p_{0}=\beta\left(x_{0}+x_{0}^{R} H_{0}^{R}\right) e\right.$, $\left\{\begin{array}{l}p_{k}=\beta x_{0} e+\beta \sum_{j=1}^{k} x_{j}^{S} H_{k-j}^{S} e+\beta \sum_{j=0}^{k} x_{j}^{R} H_{k-j}^{R} e, \quad k \geq 1 .\end{array}\right.$

We note that these results are consistent with the known results in [5] and [13].

\section{Numerical examples}

In this section, we discuss some interesting numerical examples that qualitatively describe the performance of the queueing model under study. The following examples are illustrated using the results of section 3. The algorithms have been written into a MATLAB program. For the purpose of a numerical illustration, we assume that all distribution functions in this paper are exponential, i.e. $B_{S}(x), B_{V}(x), B_{R}(x)$ are exponential distribution functions and their parameters are $\mu_{S}=2.158, \mu_{V}, \mu_{R}$ respectively. Also, we vary values of $\mu_{V}, \mu_{R}$ such that the system is stable. Numerical results are presented in Figures 1-4.

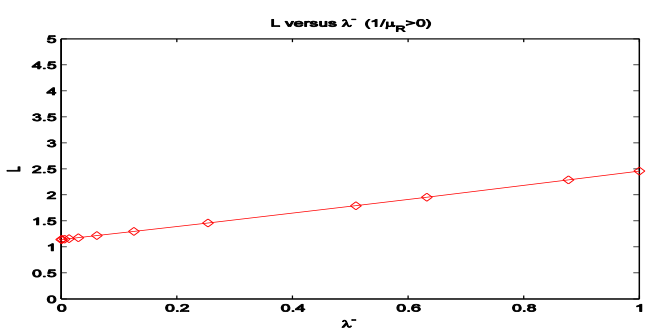

Figure 1: The mean system size versus $\lambda^{-}$with $\left(\mu_{V}, \mu_{R}\right)=(1.603,0.911)$.

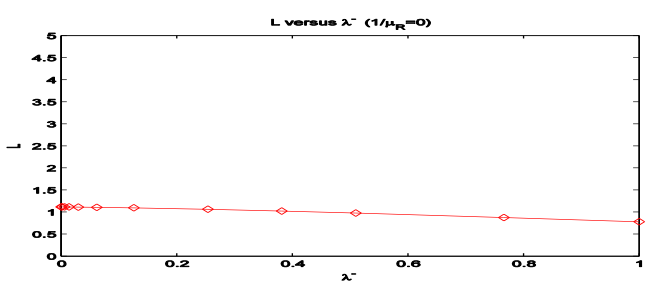

Figure 2: The mean system size versus $\lambda^{-}$with $\left(\mu_{V}, 1 / \mu_{R}\right)=(1.603,0)$.

Here we choose the following arbitrary values:

$C_{1}=\left(\begin{array}{cc}-0.7 & 0.2 \\ 0.3 & -1.4\end{array}\right), \quad D_{1}=\left(\begin{array}{cc}0.4 & 0.1 \\ 0.2 & 0.9\end{array}\right)$,

$C_{2}=\lambda^{-}\left(\begin{array}{cc}-0.007 & 0.002 \\ 0.03 & -0.009\end{array}\right), \quad D_{2}=\lambda^{-}\left(\begin{array}{ll}0.001 & 0.004 \\ 0.005 & 0.001\end{array}\right)$.

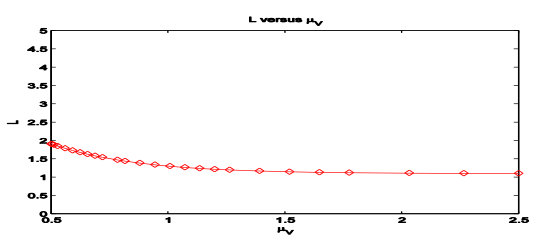

Figure 3: The mean system size versus $\mu_{V}$ with $\left(\lambda^{-}, \mu_{R}\right)=(0.212,0.911)$.

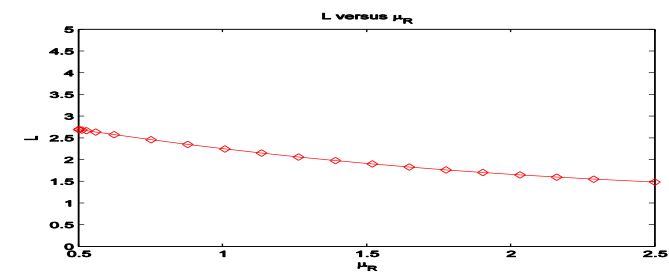

Figure 4: The mean system size versus $\mu_{R}$ with $\left(\lambda^{-}, \mu_{V}\right)=(0.212,1.603)$.

So the stationary arrival rates of the positive customers and the negative customers are $\lambda_{1}=0.7250$ and $\lambda_{2}=0.0054 \lambda^{-}$.

In Figures 1 and 2, the mean number of customers in the system is plotted against the parameter $\lambda^{-}$with $\mu_{R}=0.911$ and $1 / \mu_{R}=0$ respectively. We observe that the mean number of customers in the system increases monotonously as the value $\lambda^{-}$increases when $1 / \mu_{R}>0$ , and decreases monotonously as the value $\lambda^{-}$increases when $1 / \mu_{R}=0$. It is easily explained taking into account the fact that a negative customer not only removes the positive customer being in service but also causes the server breakdown. When the server is reliable, i.e. $1 / \mu_{R}=0$, the removal of the customer being in service can shorten the queue length. We show in Figures 3 and 4, the influence of the parameters $\mu_{V}$ and $\mu_{R}$ on the mean number of customers $L$ in the system. As is to be expected, ${ }^{L}$ decreases for increasing values $\mu_{V}$ and $\mu_{R}$.

\section{Conclusions}

This paper analyzes a MAP/ G / 1 queuing system with negative customer arrival, unreliable server and multiple vacations. By using the supplementary variables method and the censoring technique,we obtain the queue length distributions in steady state. We derive the mean of the busy period based on the renewal theory. Compared to the related work, when threre are no vacations, our results are consistent with the results in [5] and when there are no negative customers, our results are agree with the results in [13]. Hence, our model covers the models considered in [5] and [13]. This queuing system can be applied to the 
virtual channel of ATM network Performance analysis is more practical, real and reasonable.

\section{Acknowledgement}

This work is supported by Provincial Natural Science Foundation of Hunan under Grant 2019JJ50677 and the Program of Hehua Excellent Young Talents of Changsha Normal University and nurturing program of Changsha Normal University.

\section{References}

[1] Gelenbe E (1991). Product-form queueing networks with negatived and positive customers. Journal of Applied Probability, pp. 656-663. https://doi.org/10.1017/s0021900200042492

[2] Gelenbe E, Glynn P, Sigman K (1991). Queues with negative arrivals. Journal of Applied Probability, pp. 245-250.

https://doi.org/10.1017/s0021900200039589

[3] Boucherie RJ, Boxma OJ (1995). The workload in the M/G/1 queue with work removal. Probability in the Engineering and Informational Sciences, pp. 261-277. https://doi.org/10.1017/S0269964800004320

[4] Harrison PG, Patel NM, Pitel E (2000). Reliability modelling using G-queues. European Journal of Operational Research, pp. 273-287.

[5] Li QL, Zhao YQ (2004). A MAP/G/1 Queue with Negative Customers. Queueing Systems, pp. 5-43. https://doi.org/10.1023/B:QUES.0000032798.6585 8.19

[6] Cao J, Cheng K (1982). Analysis of M/G/1 queueing system with repairable service station. Acta Mathematicae Applicatae Sinica, pp. 113-127. https://doi.org/10.1007/BF01149327

[7] Neuts MF, Lucantoni DM (1979). A Markovian queue with $\mathrm{N}$ servers subject to breakdowns and repairs. Managment Science, pp. 849-861. https://doi.org/10.1287/mnsc.25.9.849

[8] Wang J, Cao J, Li Q (2001). Reliability analysis of the retrial queue with server breakdowns and repairs. Queueing Systems, pp. 363-380. https://doi.org/10.1023/a:1010918926884

[9] Harrison PG, Patel NM, Pitel E (2000). Reliability modelling using G-queues, European Journal of Operational Research, pp. 273-287.

[10] Li QL, Ying Y, Zhao YQ (2006). A BMAP/G/1 retrial queue with a server subject to breakdowns and repairs. Annals of Operations Research, pp. 233-270.

[11] Doshi BT (1986). Queueing Systems with Vacations: Survey. Queueing Systems, pp. 29-66.

[12] Sikdar K, Gupta UC (2005). The Queue Length Distributions in the Finite Buffer Bulk-Service MAP/G/1 Queue with Multiple Vacations. Top, pp. 75-103. https://doi.org/10.1007/bf02578989

[13] Kasahara S, Takine T, Hasegawa T (1996). MAP/G/1 Queues Under N-Policy With and Without
Vacations, Journal of the Operations Research Society of Japan, pp. 188-212.

[14] Bocharov PP, D’Apice C, Pechinkin AV, Salerno S (2004) Queueing Theory, Boston: Utrecht. 